\title{
Anxiety-Related Difficulties With Complex Arithmetic
}

\section{A Web-Based Replication of the Anxiety-Complexity Effect}

\author{
Julia F. Huber ${ }^{1}$ and Christina Artemenko $0^{1,2}$ \\ ${ }^{1}$ Department of Psychology, University of Tuebingen, Germany \\ ${ }^{2}$ LEAD Graduate School and Research Network, University of Tuebingen, Germany
}

\begin{abstract}
Human behavior depends on the interplay between cognition and emotion. Negative emotions like anxiety affect performance, particularly in complex tasks, by limiting cognitive resources - known as the anxiety-complexity effect. This study set out to replicate the anxiety-complexity effect in a web-based experiment. We investigated individual differences in math anxiety - a negative emotional response specific to math - and arithmetic performance $(N=382)$. The mental arithmetic task consisted of a two-digit addition and subtraction, with/ without carrying or borrowing, respectively. As expected and preregistered, higher math anxiety was related to poorer arithmetic performance, especially in complex tasks - indicating the anxiety-complexity effect. Consequently, the negative math anxiety-performance link is especially pronounced for complex arithmetic, which requires calculations across place-values and thus working memory resources. This successful replication of the anxiety-complexity effect suggests that math-anxious individuals have particular difficulties in complex arithmetic.
\end{abstract}

Keywords: anxiety-complexity effect, math anxiety, arithmetic difficulty, replication, web-based experiment

In human beings, there is a strong link between emotions, cognition, and performance. According to the processing efficiency theory (Eysenck \& Calvo, 1992), high anxiety limits working memory resources due to intrusive thoughts, and the reduced cognitive efficiency impairs task performance. This also holds for math: math anxiety, a negative emotional reaction to math, is associated with lower math performance (for the latest meta-analysis, see Barroso et al., 2021). One explanation for the math anxiety performance relation is that math anxiety preoccupies working memory, leaving fewer resources for solving the math task at hand (Ashcraft \& Krause, 2007).

Consequently, math anxiety particularly impairs performance in complex arithmetic tasks that need working memory - known as the anxiety-complexity effect (Ashcraft \& Faust, 1994). For instance, carrying (when sum of the units $>9$; e.g., $58+37$ vs. $52+43$ ) and borrowing (when unit of subtrahend > unit of minuend; e.g., 95 - 37 vs. $95-43$ ) increase the complexity of addition and subtraction, respectively (e.g., Artemenko et al., 2018). Carry and borrow problems require calculations across place-values in two-digit arithmetic (place-value computation; Nuerk et al., 2015) and rely on working memory resources (e.g., Imbo, Vandierendonck, \& De Rammelaere, 2007; Imbo, Vandierendonck, \& Vergauwe, 2007). The anxiety-complexity effect was observed for carrying in addition (Ashcraft \& Faust, 1994;
Ashcraft \& Krause, 2007; Faust et al., 1996) and might also generalize to borrowing in subtraction. Subtraction is more difficult than addition (e.g., Kong et al., 2005; Yi-Rong et al., 2011), but it is not clear whether the performance deficits related to math anxiety are dependent on the arithmetic operation.

Here we aim to replicate the anxiety-complexity effect in a preregistered web-based experiment, which has the advantage of studying individual differences in large and diverse samples (Reips, 2002). Math anxiety is expected to be negatively related to performance, especially in more complex arithmetic problems, that is, problems with as compared to without carrying and borrowing, and subtraction compared to addition (preregistered hypothesis $\mathrm{H} 4$ in https://aspredicted.org/kc5sj.pdf).

\section{Method}

\section{Participants}

For this study, German participants of a wide age range, from children in grade 3 of primary school to old age, were recruited. Out of an initial sample of $N=628$ who clicked the link, the final sample consisted of $N=382$ (129 males, 250 females, 3 other; age: $M=28.74, S D=12.84$, 
range $=8-83$ years). As preregistered (https://aspredicted. org/kc5sj.pdf), data from participants were excluded due to non-completion of the study $(n=188)$, not native German speakers $(n=12)$, younger than grade $3(n=23)$, study completion on tablet or smartphone $(n=2)$, very or extremely noisy environment during study completion $(n=5)$, responding dishonestly $(n=6)$, and accuracy below $66 \%$ ( $n=10$; cf. Artemenko et al., in press). Note that the preregistered exclusion criterion "accuracy below 33\%" (below instead of above the chance level of $50 \%$ ) should have read "accuracy below 66\%"; the criterion was changed to this, as had been originally intended.

\section{Material}

The arithmetic task included the factors operation (addition vs. subtraction) and complexity (simple = without carry/ borrow vs. complex $=$ with carry/borrow). Each condition consisted of 48 arithmetic problems with 2 two-digit operands and a two-digit solution (open material: https://osf. io/3mdb2). The arithmetic task was presented in a twoalternative choice-reaction paradigm (Figure 1, open material, https://osf.io/3mdb2). The participants were asked to mentally solve each arithmetic problem and choose the correct solution among the two options (target and distractor) by pressing the left or right arrow key with the corresponding index finger. The distractor differed at the unit $( \pm 2)$ or decade $( \pm 10)$ position from the target. Each trial started with a fixation circle at the position of the operation (duration: $500 \mathrm{~ms}$ ) and continued with the arithmetic problem until button press (time limit: $10 \mathrm{~s}$ ). The experiment was preceded by four practice items, including feedback.

Math anxiety was assessed by the abbreviated math anxiety scale (AMAS; Hopko et al., 2003) and the modified abbreviated math anxiety scale (mAMAS; Carey et al., 2017) translated to German (AMAS: Artemenko et al., in press). In each questionnaire, participants indicated on a 5-point Likert scale $(1=$ low anxiety, $5=$ high anxiety $)$ how anxious they would feel in 9 math situations described (open material: https://osf.io/3mdb2). The resulting sum scores indicate increased math anxiety by higher values. The AMAS is an appropriate instrument for the assessment of math anxiety in web-based studies (Cipora et al., 2018). The internal consistency was very good with $\alpha=.90$ (AMAS) and $\alpha=.92$ (mAMAS), comparable to the original English forms (AMAS: $\alpha=.90$, Hopko et al., 2003; mAMAS: $\alpha=.85$, Carey et al., 2017). As preregistered (https://aspredicted.org/kc5sj.pdf), all analyses were conducted with mAMAS because it focuses is on arithmetic, and it is appropriate even for children. Moreover, AMAS and mAMAS were highly correlated $(r=.949)$.

\section{Procedure}

The web-based experiment was programmed with jsPsych (Version 6.1.0; De Leeuw, 2015) and conducted on Pavlovia (pavlovia.org). All texts and numbers were presented in white against a black background. Because of time restrictions in web-based experiments, the stimulus sets were divided equally into four blocks (with 12 items per condition); each participant completed two of four blocks with randomly presented stimuli and a break between the blocks. The experimental task of arithmetic performance was administered before the math anxiety questionnaires AMAS and mAMAS. In the end, quality items regarding noise, honesty, and device (open material: https://osf.io/ $3 \mathrm{mdb} 2$ ) were used to ensure the data quality of less controllable web-based experiments (Reips, 2002). The total duration of the experiment was about 15-20 min.

\section{Analysis}

The preregistered analyses [https://aspredicted.org/kc5sj. pdf] were conducted on the RT data (open data and analysis: https://osf.io/3mdb2) using R (R Core Team, 2020). First, we removed (1) incorrectly solved trials (errors and missings, 9.07\%), (2) RTs below 200 ms (anticipations, $0.04 \%$ ), and (3) RTs deviating more than $3 S D$ from the individual $M$ (iterative trimming procedure, which took six iterations, $0.93 \%$ ).

RTs were analyzed using linear mixed effects (LME) models. We started with the full model:

$$
\begin{aligned}
y_{i j}= & \beta_{0}+\beta_{1} \text { operation }+\beta_{2} \text { complexity } \\
& +\beta_{3} \text { math anxiety } \\
& +\beta_{4}(\text { operation } \times \text { complexity }) \\
& +\beta_{5}(\text { operation } \times \text { math anxiety }) \\
& +\beta_{6}(\text { complexity } \times \text { math anxiety }) \\
& +\beta_{7}(\text { operation } \times \text { complexity } \times \text { math anxiety }) \\
& +v_{0 i}+v_{1 i} \text { operation }+v_{2 i} \text { complexity } \\
& +v_{3 i}(\text { operation } \times \text { complexity })+w_{0 j},
\end{aligned}
$$

with $\beta_{\mathrm{O}}$ indicating the intercept, $i$ a specific participant, and $j$ a specific item. The categorical predictors operation and complexity were effect-coded: -0.5 for addition, +0.5 for subtraction and -0.5 for simple, +0.5 for complex. The continuous predictor math anxiety was centered. The model included a random intercept for participants $\left(v_{0 i}\right)$ and items $\left(w_{0 j}\right)$, and random slopes for operation $\left(v_{1 i}\right)$, complexity $\left(v_{2 i}\right)$, and their interaction $\left(v_{3 i}\right)$. In a backward selection procedure (function step of the package lmerTest; Kuznetsova et al., 2017), random effects were determined by likelihood ratio tests favoring the simpler 
Table 1. Fixed effects of the reduced model

\begin{tabular}{|c|c|c|c|c|c|c|}
\hline Fixed effect & Estimate (ms) & SE (ms) & $d f$ & $t$ & $p$ & Adjusted $p$ \\
\hline Intercept & $4,022.83$ & 56.43 & 552.95 & 71.29 & $<.001$ & $<.001$ \\
\hline Operation & 669.55 & 68.16 & 224.39 & 9.82 & $<.001$ & $<.001$ \\
\hline Complexity & 897.29 & 67.39 & 215.05 & 13.32 & $<.001$ & $<.001$ \\
\hline Math Anxiety & 14.29 & 5.55 & 380.95 & 2.58 & .010 & .011 \\
\hline Complexity $\times$ Math Anxiety & 6.88 & 2.69 & 379.91 & 2.56 & .011 & .011 \\
\hline
\end{tabular}

Note. Operation was effect-coded with -0.5 for addition and +0.5 for subtraction and complexity with -0.5 for simple and +0.5 for complex problems. Math anxiety was centered. $p$-values were FDR-adjusted.

model, when $\alpha>.1$, and fixed effects by $F$ - and $t$-tests favoring the simpler model, when $\alpha>.05$.

This procedure resulted in the following reduced model:

$$
\begin{aligned}
y_{i j}= & \beta_{0}+\beta_{1} \text { operation }+\beta_{2} \text { complexity } \\
& +\beta_{3} \text { math anxiety } \\
& +\beta_{6}(\text { complexity } \times \text { math anxiety })+v_{0 i} \\
& +v_{1 i} \text { operation }+v_{2 i} \text { complexity } \\
& +v_{3 i}(\text { operation } \times \text { complexity })+w_{0 j},
\end{aligned}
$$

$p$-values were calculated using the Satterthwaite's method and adjusted for multiple testing using the false discovery rate (FDR). As preregistered, we considered a possible speed-accuracy trade-off by calculating the correlation between RT and accuracy and further conducted an extreme group analysis (see open material, https://osf. io/3mdb2).

\section{Results}

The results of the RT analysis are given in Table 1 . The anxiety-complexity effect was found, as the significant interaction between complexity and math anxiety indicated that the math anxiety slope for complex problems (estimate $=$ $17.73 \mathrm{~ms}, S E=6.29 \mathrm{~ms}$ ) was steeper than for simple problems (estimate $=10.86 \mathrm{~ms}, S E=5.06 \mathrm{~ms}$; see Figure 1). The math anxiety effect indicated that the higher the math anxiety level, the longer the participants needed to solve the arithmetic problems. The complexity effect indicated that for an average math anxiety level of the sample $(M=$ $19.37, S D=7.86$ ), solving simple problems (estimate $=$ $3,574 \mathrm{~ms}, S E=62 \mathrm{~ms}$ ) was faster than solving complex problems (estimate $=4,471 \mathrm{~ms}, S E=69 \mathrm{~ms}$ ). The operation effect indicated that participants solved addition problems faster (estimate $=3,688 \mathrm{~ms}, S E=63 \mathrm{~ms}$ ) than subtraction problems (estimate $=4,358 \mathrm{~ms}, S E=68 \mathrm{~ms}$ ).

Finally, a significant negative correlation between mean RT and mean accuracy per participant, $r(380)=-.35$, $p<.001$, indicated that participants who responded faster were also more accurate (i.e., the opposite of a speedaccuracy trade-off).

\section{Discussion}

The purpose of this study was to investigate whether the anxiety-complexity effect can be replicated in a preregistered web-based experiment. As expected, higher math anxiety was associated with poorer arithmetic performance, especially with carrying and subtraction with borrowing. While the anxiety-complexity effect was successfully replicated (e.g., Ashcraft \& Faust, 1994), we did not find evidence for operation-specific effects.

These results allow for conclusions about the cognitive processes underlying the relation between math anxiety and arithmetic performance. Replicating previous studies (Ashcraft \& Faust, 1994; Ashcraft \& Krause, 2007; Faust et al., 1996), math anxiety was negatively related to performance, especially in complex arithmetic requiring placevalue computation as carrying in addition and borrowing in subtraction. Thus, the anxiety-complexity effect is a robust finding that can be found even for brief assessments and strictly controlled stimulus material (e.g., problem size).

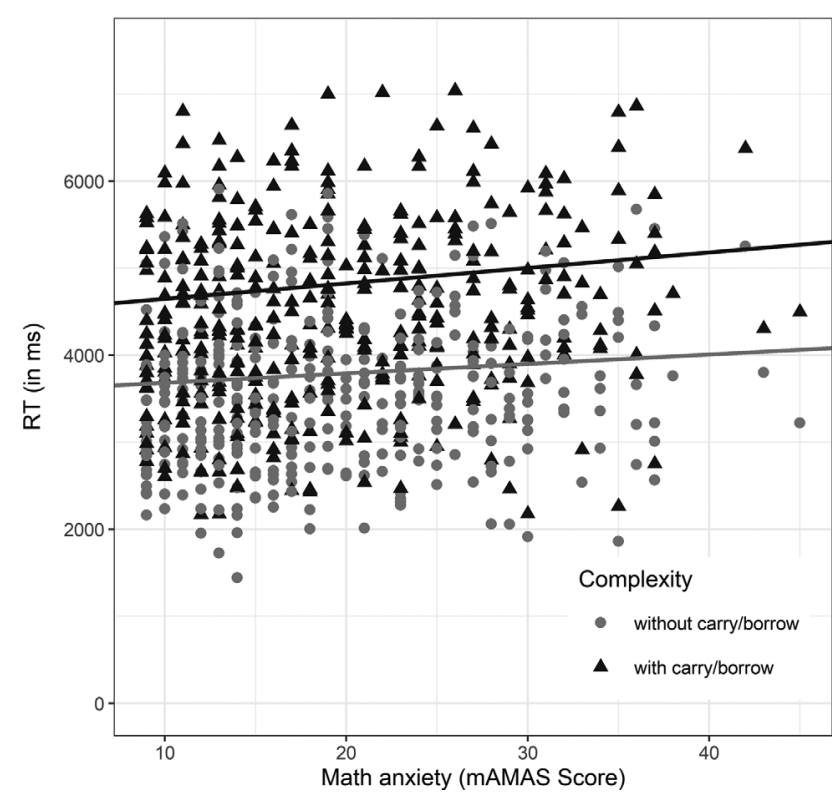

Figure 1. Anxiety-complexity effect: Higher math anxiety is related to a larger difference in RT between simple and complex arithmetic problems. 
Due to anxiety-related worries, math anxiety might reduce working memory resources, which are particularly involved in carrying and borrowing (Imbo, Vandierendonck, \& De Rammelaere, 2007; Imbo, Vandierendonck, \& Vergauwe, 2007), and, therefore, might reduce arithmetic performance (Ashcraft \& Krause, 2007).

Although our results confirm that subtraction was more difficult than addition (see also Kong et al., 2005), we did not find a differential effect of math anxiety for addition and subtraction problems. Because there is no evidence that the strategies for these arithmetic operations differ in working memory load (cf. Imbo \& Vandierendonck, 2007), working memory may play a crucial role in the anxietycomplexity effect (see also Ashcraft \& Krause, 2007). In conclusion, the math-related deficit in individuals with math anxiety is larger for complex arithmetic only if the demand on working memory is higher (as for place-value computation), independent of the arithmetic operation. Note that our findings for addition and subtraction were not operation-specific, and the investigation of math anxiety might extend to other arithmetic operations in the future.

\section{Conclusion}

This replication of the anxiety-complexity effect in an online setting shows that web-based experiments can be used in this area of psychological research. Web-based data collection provides a large, heterogeneous sample, which allows for higher generalizability and external validity. A specific problem in math anxiety research is a selection bias, as avoidance of math-related activities is part of the anxiety reaction (e.g., Ashcraft \& Faust, 1994). Web-based research can reduce the inhibition to participate due to the anonymous setting (Joinson, 2001). Although the study indeed recruited a heterogeneous sample regarding age and math anxiety, most subjects nevertheless consisted of young adults and individuals with low math anxiety. The web-based setting provides future opportunities for crosscultural studies to generalize our results. A major limitation of our findings is that the quasi-experimental design does not allow us to draw causal inferences, such as that math anxiety impairs performance in complex arithmetic. Our research on the anxiety-complexity effect is further restricted to arithmetic performance; future research might investigate more complex math tasks, such as fractions and word problems.

\section{References}

Artemenko, C., Masson, N., Georges, C., Nuerk, H.-C., \& Cipora, K. (in press). Not all elementary school teachers are scared of math. Journal of Numerical Cognition. https://psyarxiv.com/ wej8z
Artemenko, C., Soltanlou, M., Dresler, T., Ehlis, A.-C., \& Nuerk, H. C. (2018). The neural correlates of arithmetic difficulty depend on mathematical ability: Evidence from combined fNIRS and ERP. Brain Structure and Function, 223(6), 25612574. https://doi.org/10.1007/s00429-018-1618-0

Ashcraft, M. H., \& Faust, M. W. (1994). Mathematics anxiety and mental arithmetic performance: An exploratory investigation. Cognition \& Emotion, 8(2), 97-125. https://doi.org/10.1080/ 02699939408408931

Ashcraft, M. H., \& Krause, J. (2007). Working memory, math performance, and math anxiety. Psychonomic Bulletin \& Review, 14(2), 243-248. https://doi.org/10.3758/BF03194059

Barroso, C., Ganlex, C. M., McGraw, A. L., Geer, E. A., Hart, S. A., \& Daucourt, M. C. (2021). A meta-analysis of the relation between math anxiety and math achievement. Psychological Bulletin, 147(2), 134-168. https://doi.org/10.1037/bul0000307

Carey, E., Hill, F., Devine, A., \& Szucs, D. (2017). The Modified Abbreviated Math Anxiety Scale: A valid and reliable instrument for use with children. Frontiers in Psychology, 8, 1-13. https:// doi.org/10.3389/fpsyg.2017.00011

Cipora, K., Willmes, K., Szwarc, A., \& Nuerk, H.-C. (2018). Norms and validation of the online and paper-and-pencil versions of the Abbreviated Math Anxiety Scale (AMAS) for Polish adolescents and adults. Journal of Numerical Cognition, 3(3), 667693. https://doi.org/10.5964/jnc.v3i3.121

De Leeuw, J. R. (2015). jsPsych: A JavaScript library for creating behavioral experiments in a Web browser. Behavior Research Methods, 47(1), 1-12. https://doi.org/10.3758/s13428-014-0458-y

Eysenck, M. W., \& Calvo, M. G. (1992). Anxiety and performance: The processing efficiency theory. Cognition \& Emotion, 6(6), 409-434. https://doi.org/10.1080/02699939208409696

Faust, M., Ashcraft, M. H., \& Fleck, D. (1996). Mathematics anxiety effects in simple and complex addition. Mathematical Cognition, 2(1), 25-62. https://doi.org/10.1080/135467996387534

Hopko, D., Mahadevan, R., Bare, R., \& Hunt, M. (2003). The Abbreviated Math Anxiety Scale (AMAS): Construction, validity, and reliability. Assessment, 10(2), 178-182. https://doi.org/ $10.1177 / 1073191103010002008$

Imbo, I., \& Vandierendonck, A. (2007). The role of phonological and executive working memory resources in simple arithmetic strategies. European Journal of Cognitive Psychology, 19(6), 910-933. https://doi.org/10.1080/09541440601051571

Imbo, I., Vandierendonck, A., \& De Rammelaere, S. (2007). The role of working memory in the carry operation of mental arithmetic: Number and value of the carry. The Quarterly Journal of Experimental Psychology, 60(5), 708-731. https://doi.org/ 10.1080/17470210600762447

Imbo, I., Vandierendonck, A., \& Vergauwe, E. (2007). The role of working memory in carrying and borrowing. Psychological Research, 71(4), 467-483. https://doi.org/10.1007/s00426-006-0044-8

Joinson, A. (2001). Self-disclosure in computer-mediated communication: The role of self-awareness and visual anonymity. European Journal of Social Psychology, 31(2), 177-192. https:// doi.org/10.1002/ejsp.36

Kong, J., Wang, C., Kwong, K., Vangel, M., Chua, E., \& Gollub, R. (2005). The neural substrate of arithmetic operations and procedure complexity. Cognitive Brain Research, 22(3), 397405. https://doi.org/10.1016/j.cogbrainres.2004.09.011

Kuznetsova, A., Brockhoff, P. B., \& Christensen, R. H. B. (2017). ImerTest Package: Tests in linear mixed effects models. Journal of Statistical Software, 82(13), 1-26. https://doi.org/ 10.18637/jss.v082.i13

Nuerk, H.-C., Moeller, K., \& Willmes, K. (2015). Multi-digit number processing. In R. Cohen Kadosh \& A. Dowker (Eds.), Oxford handbook of mathematical cognition (pp. 106-139). Oxford University Press. 
R Core Team. (2020). R: A language and environment for statistical computing (Version 4.0.3). R Foundation for Statistical Computing. https://www.r-project.org/

Reips, U.-D. (2002). Standards for Internet-based experimenting. Experimental Psychology, 49(4), 243-256. https://doi.org/ 10.1026//1618-3169.49.4.243

Yi-Rong, N., Si-Yun, S., Zhou-Yi, G., Si-Run, L., Yun, B., Song-Hao, L., \& Chan, W. Y. (2011). Dissociated brain organization for twodigit addition and subtraction: An fMRI investigation. Brain Research Bulletin, 86, 395-402. https://doi.org/10.1016/j. brainresbull.2011.08.016

\section{History}

Received February 1, 2021

Revision received July 29, 2021

Accepted July 29, 2021

Published online December 17, 2021

\section{Acknowledgments}

We thank Natascha Klonsdorf, Yvonne Beck, Steffen Tietz, and Morgan Hess.

\section{Publication Ethics}

The study was approved by the Ethics Committee for Psychological Research of the University of Tuebingen, and all participants provided informed consent via mouse click.

\section{Open Data}

Data, analysis scripts, and materials can be accessed at the Open Science Framework (OSF; https://osf.io/3mdb2).

\section{Funding}

This research was supported by: Tuebingen Postdoc Academy for Research on Education; Ministry of Science, Research and the Arts Baden-Wuerttemberg; European Social Fund; LEAD Graduate School \& Research Network [GSC1028]; German Research Foundation (DFG; NU265/5-1). Open access publication enabled by the University of Tübingen.

\section{Christina Artemenko}

Department of Psychology

University of Tuebingen

Schleichstr. 4

72076 Tuebingen

Germany

christina.artemenko@uni-tuebingen.de 